\title{
Extending shelf life of fruits by using some microorganisms biological products
}

\begin{abstract}
softening was considered a main problem in fresh fruits and vegetables during marketing from farm to consumer, that occur as a natural process of maturation and water loss which lead to making them loss of economic value, shortening of shelf life and undesirable for consumed by consumers, therefore, it became important to find some new technique to save these fruits and vegetables, so, pullulans witch know as a neutral water soluble polysaccharide that produced from starch by the fungus Aureobasidium pullulans by fermentation and killer toxin witch know a neutral protein that produced by Yeast Saccharomyces cerevisiae (Bakery yeast) were use as a safe biological materials in this case to inhibition microorganisms growth and weight loss and spoilage to extending shelf life of fruits and vegetables by using some biotechnological methods and biological products from some microorganisms.
\end{abstract}

Keywords: pullulan, killer toxin, bakery yeast, Saccharomyces cerevisiae, Aureobasidium pullulans, microorganisms growth, weight loss, spoilage, fruits, extending shelf life
Volume 2 Issue 5 - 2017

\author{
Mohammed A. Alsoufi,' Raghad A Aziz ${ }^{2}$ \\ 'Market Research and Consumer Protection Center, University \\ of Baghdad, Iraq \\ ${ }^{2}$ Department of Science, University of Al-Mustansiriah, Iraq
}

Correspondence: Mohammed A Alsoufi, Market Research and Consumer Protection Center, University of Baghdad, Iraq, Tel 00964 77026/2928, Email alsoufim@yahoo.com

Received: June 05, 2017 | Published: July 24, 2017

\section{Introduction}

In recent years; the increased demand of fresh foods led to the search and finding new method which ensures to keep foods, with freshness and high quality. ${ }^{1}$ Increasing consumer demand for extended plant material storage duration has mobilized the food industry to search for methods to maintain raw material quality and protection of a wide group of food products. ${ }^{2}$ All fruits and vegetables are harvested when they are completely ripe, the main problem with them it continues to ripen while they are being stored because it causes irreversible wilt and weight loss which results to shortened shelf-life and loss of economic and commercial value, so applying edible coatings that defined as thin layers of biomaterials, which form a barrier around products in the form of tightly adhering coating, and may concurrently be consumed together with the protected food, this edible coatings manufacture from natural materials like polysaccharides, proteins and lipids might be one of the effective methods to prolong shelf-life stability at storage in the room temperature for these types of foods by slows down gases exchange, controls respiration process, limits mass loss and R.H. transmission and decrease in microorganisms growth on the surface. ${ }^{3-5}$

\section{Type of biological products}

\section{Pullulan}

Pullulan $\left(\mathrm{C}_{6} \mathrm{H}_{10} \mathrm{O}_{5}\right) \mathrm{H}_{2} \mathrm{O}$ is a neutral, water-soluble polysaccharide produced from starch by the fungus Aureobasidium pullulans, by means of a process of fermentation. Chemically, this polymer consists of $\alpha-1,6$ linked maltotriose residues, which in turn are composed of three glucose molecules connected to each other by $\alpha-1,4$ glycosidic bonds. ${ }^{6,7}$

Pullulan characterized by nontoxic for human and animal, low caloric and totally biodegradable, have good adhesive characteristics that enables it to be applied for coating of food products, colorless, odorless, no taste, can be very thin and have a big barrier characteristics in relation between oxygen and carbon dioxide, which had effect upon growth inhibition of most of the tested microorganisms, responsible for decay of food, so it can be an useful element to ensure microbiological safety of food. ${ }^{8}$ This polysaccharide is of economic importance with increased application in food, pharmaceutical, agricultural and chemical industries. ${ }^{9}$ Pullulan produces a high viscosity solution at a relatively low concentration and can be used for oxygen impermeable films and fibers, thickening or extending agents, or adhesive or encapsulating agents, despite being a $\alpha$-D-glucan, Pullulan is resistant to $\alpha$-D-amylolysis and may be used in low-calorie food formulation. ${ }^{10}$ The use of Pullulan in biomedical field is increasing contemporarily due to its non toxic, non immunogenic, biocompatible and inert nature. Pullulan as a carrier for drug delivery, ${ }^{11}$ tissue engineering and grafting, ${ }^{12}$ liver targeting of drug loaded pullulans, ${ }^{13}$ anti cancer drug, ${ }^{14}$ as drug delivery systems, particularly in the form of micro gels and nano gels, ${ }^{15}$ gene delivery, ${ }^{16}$ bio imaging by nanoparticles shell. ${ }^{17}$ Pullulan is also being used for the production of biodegradable plastics in Japan and U.S.A., because it resembles polyethylene with properties like tensile strength and ability to form thin transparent oxygen impermeable films. ${ }^{18}$

Pullulan produced by the fungus Aureobasidium pullulans, by means of a process of fermentation. Aureobasidium pullulans (De Bary) G. Around is a ubiquitous, polymorphic and oligotrophe black yeast like micro fungus that occurs frequently in wide range of tropical and temperate environment with fluctuating moisture content in phyllosphere, and also isolated from damp indoor surfaces, food and feed substances, it has also been found in the osmotically stressed environments like hyper saline water in salterns and the rocks. ${ }^{3,19}$

\section{Killer toxin}

Yeast Saccharomyces cerevisiae (Bakery yeast) have the ability to produce of some types of proteins which working for prevent or delay the growth of other microorganisms in the same growth medium and these proteins hers acidic nature and specific reaction which depend of some environmental factors such as $\mathrm{pH}$, temperature and ventilation. ${ }^{20}$ Moreover, the yeast has many mechanisms that explain their ability to neutralizing some of microorganisms through the directly influence, or by inhibition of some pathogens, contribution to stimulating the 
immune system and prevent bacterial toxins from access to receptors in the cells. ${ }^{21}$ So, yeast widely used instead of antibody for the ability to prevent pathogenic bacteria of colonize the gastrointestinal area through competition for nutrients, which prevents or reduces effect these microorganisms, ${ }^{22}$ several studies proved that yeast hers an important role in providing immune protection against pathogenic infections of the digestive tract by stimulating the increase production of immunoglobulin IgA as well as work on organize of the intestinal environment and reduce division of cells pathogens, ${ }^{23}$ as it proved high efficiency in the treatment of acute and chronic diarrhea which caused by intestinal bacteria which endemic to intestines of infected adults and children, ${ }^{24}$ as well used to prevent diarrhea for the traveler's and relieve abdominal pain of enteric nervous, ${ }^{25}$ likewise used as therapy for children who suffer from Candidiasis disease which caused by $C$. Albicans. ${ }^{26}$

\section{Applications}

\section{Effect of microorganism's growth}

Many research refer to inhibitory effect for killer toxin which purification from bakery yeast and pullulans that production from locally isolate Aureobasidium pullulans on bacteria, mold and yeast, Alsoufi \& Aziz ${ }^{1}$ found that inhibition of E. Coli, S. Typhimurium, Aspergillus ssp., Penicillium ssp., C. Albicans, P. Jadinii, was 71, 62, $52,59,68$ and $76 \%$ respectively, at a rate of $64.6 \%$ for killer toxin, and was $68,70,42,48,61$ and $63 \%$ respectively, at a rate of $58.6 \%$ for Pullulan, and 74, 68, 65, 68, 71 and $73 \%$ respectively, at a rate of $69.83 \%$ for $25 \%$ Pullulan $+75 \%$ killer toxin, and was $83,76,72,81$, 76 and $87 \%$ respectively, at a rate of $79.16 \%$ for $50 \%$ Pullulan $+50 \%$ killer toxin, and was $67,72,54,56,64$ and $69 \%$ respectively, at a rate of $63.66 \%$ for $75 \%$ Pullulan $+25 \%$ killer toxin; while, ChlebowskaSmigiel \& Gniewosz ${ }^{8}$ studied effect of pullulans coating as antimicrobial activity on moulds, yeasts and bacterial growth, they found that the inhibition of pullulans was $100 \%$ on bacterial species such as Citrobacter freundii ATCC 8090, Lactobacillus plantarum ATCC 4080, and Pseudomonas fluorescens, while the inhibition was $80 \%$ on Lactobacillus brevis. The inhibition of pullulans was $70 \%$ on Escherichia coli ATCC 25922 and Tetracoccus sp. and 60\% on Micro-coccus luteus ATCC 9341 and Bacillus subtilis ATCC 6650, They suggest that pullulans coating can be a useful application to ensure food safety and quality from microbiological deterioration, because it had important effect on relation between oxygen and carbon dioxide in microorganisms. In the same context, Gniewosz, et al. ${ }^{5}$ was studied antimicrobial activity of pullulans film incorporated with meadowsweet flower extracts (Filipendulae ulmariae flos) on postharvest quality of apples, They found that inhibition of pullulans on Staphylococcus aureus ATCC 25923, Bacillus subtilis ATCC 6633, Salmonella enteritidis ATCC 13076 and Escherichia coli ATCC 25922 was more than degree of mold species such as Penicillium expansum ATCC 7861, Rhizopus arrhizus ATCC 11145 and Aspergillus niger ATCC 9142, also they refer that gram-positive bacteria like S. Aureus \& B. Subtilis were demonstrated higher resistance (MIC 0.6-6.0mg/ml), also Krasniewska, et al. ${ }^{2}$ was used Pullulan coating that enriched with plant extracts from Satureja hortensis L. in different concentrations to maintain pepper and apple quality and safety and they obtained that the inhibition zone was significantly increased $(p<0.05)$ for all the tested strains as the concentrations of extracts in the pullulans film, in $5 \%$ addition of extract to the pullulans film inhibited the growth of gram-positive bacteria, while gram-negative bacteria were inhibited by $20 \%$ in the film, they explain that, the gram-negative bacteria are more resistant to antimicrobial compounds than gram-positive bacteria and this fact may be related to the structure of the cell wall of gram-negative bacteria which is characterized by the presence of an additional external lipopolysaccharide (LPS) membrane, whilst, Kanmani \& $\mathrm{Lim}^{27}$ were refer to antimicrobial activities for pullulansmediated silver nanoparticles against some microorganisms that causes food-born and multidrug resistant pathogens, they found that all bacterial pathogens were highly inhibited in a dose-dependent manner, increases in the inhibition zones were observed with an increase in the amount of AgNPs. The bacterial pathogens like Pseudomonas. Aeruginosa was more susceptible to AgNPs followed by Klebsiella. Pneumoniae and E. Coli, the food-born pathogen Listeria. monocytogenes was less susceptible to the AgNPs, overall, the gram negative bacterial pathogens were highly suppressed by the AgNPs compared to gram positive bacterial pathogens, that probably due to the thick cell wall of gram positive bacteria, which are generally composed of a three-dimensional thick peptidoglycan (PG 20-80nm) layer compared to that of gram negative bacteria $(\sim 7-8 \mathrm{~nm})$, the PG layer possesses linear polysaccharide chains cross linked by more short peptides and thus forms a complex structure which makes it difficult for AgNPs to penetrate gram positive bacteria, also Jittinan, et al., ${ }^{28}$ was observe that oral film of herbal extract which contain different concentration of pullulans showed antimicrobial activity against Streptococcus mutans, Streptococcus sanguis and Porphyromonas gingivalis, while Wagner, et al. ${ }^{29}$ explain that A. pullulans solution with different concentrations was very active against grey mold Botrytis cinerea and Monilinia fructigena. On the other side, Premanathan, et al. ${ }^{26}$ was refer to killer toxin form $S$. cerevisiae as a active agent for inhibition of C. Albicans and Yersinia ruckeri, also, all of Aziz, et al. ${ }^{20} \&$ Aziz, et al, ${ }^{30,31}$ observe that crude extract and purified killer toxin form $S$. Cerevisiae was active antimicrobial against $E$. Coli, Salmonella enterica, S. Typhimurium, Shigella flexneri \& Shigella sonnei, while, Alsoufi ${ }^{3}$ observed the effect coating of pullulans which production from locally isolate Aureobasidium pullulans on growth of Bacillus subtilis, Staphylococcus aureus, Escherichia coli and Salmonella typhimuram, it was $63,65,70$ and $75 \%$ for respectively.

\section{Weight loss and spoilage}

The main problem in fresh fruits and vegetables are turnover on the markets is softening that caused by the natural process of maturation and water loss, this changes are considered undesirable by consumers. ${ }^{31}$ Weight loss in fresh fruits and vegetables caused a short shelf-life and loss of economic value, additionally, this parameter has a strong impact on the appearance, due to shrinkage, both temperature and storage time contributed to increase weight loss. ${ }^{2}$ Storage at $16^{\circ} \mathrm{C}$ reported higher weight loss than storage at $6^{\circ} \mathrm{C}$, polysaccharide coatings exhibit high barrier properties toward oxygen, the rate of oxygen transfer through pullulans films is lower compared to polypropylene and polyethylene films, this enables the formation of pullulans coatings to reduce the rate of fruit and vegetable respiration. ${ }^{8}$ So many researches discuss this important parameter.

Results of Alsouf ${ }^{3}$ showed the weight loss for uncoated and coated peach with 10 and $20 \%$ pullulans water solutions which production from locally isolate Aureobasidium pullulans that storage at $25^{\circ} \mathrm{C}$ for 21 days, the percentage of weight loss of uncoated peach was $3.2,4.8$, $6.1,7,7.2,8.3$ and $9.1 \%$, Values of weight loss in coated peach with $10 \%$ pullulans were $3.1,4.5,5.8,6.2,6.8,7.9$ and $8.9 \%$, while values in coated peach with $20 \%$ pullulans were $2.9,3.8,4.1,4.6,5.8,6.4$ and $7.6 \%$ when stored for $3,6,9,12,15,18$ and 21 days respectively, while the results of weight loss for uncoated and coated peach storage at $4^{\circ} \mathrm{C}$ for 10 days showed the percentage values of weight loss for 
uncoated peach increased gradually between the first and tenth day of stored were $0.01,0.06,0.16,0.23,0.35,0.46,0.6,0.68,0.73$ and $0.75 \%$. Values of coated peach with $10 \%$ pullulans were $0.01,0.05$, $0.12,0.16,0.2,0.3,0.5,0.58,0.64$ and $0.71 \%$, while values in coated peach with $20 \%$ Pullulan were $0.015,0.02,0.04,0.08,0.1,0.12,0.18$, $0.3,0.46$ and $0.5 \%$, when samples stored for $1,2,3,4,5,6,7,8,9$ and 10days respectively. The changes in weight loss for uncoated and coated pear with 10 and $20 \%$ pullulans that stored at $25^{\circ} \mathrm{C}$ showed that the weight loss values of uncoated pear were 1.4, 2, 2.6, 4.4, 5.5, 6.4 and $7.2 \%$, the values in coated peach with $10 \%$ pullulans were $1.2,1.6,2,2.4,4.3,5.2$ and $6.6 \%$, while in coated peach with $20 \%$ pullulans were $0.8,1,1.4,1.6,3,4.6$ and $5.4 \%$, when these samples stored at 3, 6, 9, 12, 15, 18 and 21 days respectively, also, he observe that the weight loss values of uncoated pears were $0.02,0.07,0.14$, $0.16,0.3,0.33,0.44,0.45,0.52$ and $0.54 \%$ for 10 days storage at $4{ }^{\circ} \mathrm{C}$, while, the values for coated pears with $10 \%$ Pullulan were $0.02,0.06$, $0.11,0.16,0.21,0.3,0.36,0.38,0.44$ and $0.46 \%$, while coated pears with $20 \%$ pullulans were $0.01,0.05,0.08,0.09,0.1,0.16,0.19,0.24$, 0.26 and $0.28 \%$, as there samples stored for $1,2,3,4,5,6,7,8,9$ and 10days respectively. Chlebowska-Smigiel, et al ${ }^{4}$ used Pullulan coatings to prolong apples shelf-life stability by coating apples with 15 and $20 \%$ Pullulan stored at $4{ }^{\circ} \mathrm{C}$ and $22^{\circ} \mathrm{C}$ for 39 days, they noticed that the highest mass loss was with $15 \%$ pullulans coat samples during the first two days of storage and then between 18th and 22nd day at $4^{\circ} \mathrm{C}$, the coated with $20 \%$ Pullulan also the highest mass loss was observed in the first two days and then considerable fall between 13th and 18th day of storage, also they found on the following days, apples mass losses were tended to decrease independently from the Pullulan and the values of changed was from $0.49-0.58 \%$ after the first day of storage up to $0.11-0.13 \%$ after the very last day, and for the comparative apples (without Pullulan coating) the mass loss was evidently highest than for these coated and was $0.9 \%$ after first day of storage and $0.2 \%$ after the last day, and observed the mass losses for the apples covered with the 15 and $20 \%$ Pullulan were less than for the control apples (uncoated) when stored at room temperature of $22^{\circ} \mathrm{C}$ during 39day, the highest mass losses for the control apples were observed during the first 11 days of storage (and they were $1.35-0.74 \%$ ), at the same time the mass losses of the apples covered with the protecting Pullulan coatings were only $0.82-0.51 \%$, the coatings produced from either 15 or $20 \%$ Pullulan had better physical properties at $4{ }^{\circ} \mathrm{C}$ than at $22^{\circ} \mathrm{C}$. In lower temperature it was thin, shiny and smooth, at $22^{\circ} \mathrm{C}$ starting from the 5 th day of storage, became wrinkled and the fruit surface started to go off, also, Krasniewska, et al. ${ }^{2}$ use Pullulan coating that enriched with plant extracts (SH) from Satureja hortensis L. to maintain pepper and apple quality and safety, they noticed that weight loss increased significantly $(\mathrm{p}<0.05)$ during the storage of at 6 and $16^{\circ} \mathrm{C}$ for uncoated peppers compared to peppers coated with Pullulan and Pullulan (SH) extract, while water loss was observed significantly lower $(p<0.05)$ in coated samples, and throughout storage at $6^{\circ} \mathrm{C}$, the uncoated peppers and peppers coated with Pullulan and with Pullulan (SH) extract lost respectively $12.01,10.47$ and $8.87 \%$ of their initial weight at 28 days of storage, the weight loss in uncoated peppers stored at $16^{\circ} \mathrm{C}$ was the highest $12.66 \%$ at 14 day, while peppers coated with Pullulan and Pullulan (SH) extract reached lower values of 10.26 and 9.75\%, respectively, and a similar behavior was observed for apples stored at $2^{\circ} \mathrm{C}$ and $16^{\circ} \mathrm{C}$, weight loss for coated apples was significantly lower $(\mathrm{p}<0.05)$ than that of the controls, apples stored at $2^{\circ} \mathrm{C}$ showed losses of about $2.84 \%$ for uncoated samples, whereas for apples coated with Pullulan and with Pullulan (SH) extract the weight loss was 1.93 and $1.61 \%$, respectively, reductions in weight loss in apples stored at $16^{\circ} \mathrm{C}$ were $4.13,2.93$ and $2.86 \%$ respectively, for uncoated apples, those coated with Pullulan and with Pullulan (SH) extract. Whilst Gniewosz, et al. ${ }^{5}$ used Pullulan film incorporated with meadowsweet flower extracts (Filipendulae ulmariae flos) on postharvest quality of apples, they refer that the highest weight losses were noted in the samples stored at $24^{\circ} \mathrm{C}$, during the first 8 days, the losses in sample weight did not different significantly statistically, which was probably caused by rapid water evaporation from the surface (uncoated samples) and from coatings (coated samples), at the end of the storage period, weight losses about $0.5 \%$ lower were noted in the case of samples coated with Pullulan coatings with meadowsweet flower extracts (EMFs) compared to uncoated ones, the samples stored in refrigerated conditions demonstrated lower weight losses, the addition of (EMFs) to Pullulan coatings contributed to a decrease in weight loss of up to $2.5 \%$ when used Pullulan coatings with ethanol meadowsweet flower extracts (eEMF) and $2.3 \%$ with Pullulan coatings with water-ethanol mixture meadowsweet flower extracts (weEMF), however, Alsoufi \& Aziz ${ }^{1}$ observe that the percentage values of weight loss for uncoated and coated apples with $100 \%$ Killer toxin from Bakery yeast, $100 \%$ Pullulan, 50\% Pullulan $+50 \%$ Killer toxin, $25 \%$ Killer toxin $+75 \%$ Pullulan and $75 \%$ Killer toxin $+25 \%$ Pullulan solutions were 3.2, 4.8, $6.1,7,7.2,8.3$ and $9.1 \% ; 3.3,4.2,5.1,5.6,6.1,7.2$ and $8.3 \% ; 2.9$, $3.8,4.1,4.6,5.8,6.4$ and $7.6 \% ; 2.6,2.8,3.2,3.3,4,4.7$ and $5.1 \%$; $2.9,3.3,3.5,4.1,5.3,5.7$ and $6 \% ; 3,3.5,3.7,4.4,5.6,5.9$ and $6.5 \%$ respectively, when storage at $25^{\circ} \mathrm{C}$ for $3,6,9,12,15,18$ and 21 day respectively, while observe spoilage time (days) during apples storage at $25^{\circ} \mathrm{C}$ showed that the spoilage began to appear at 4, 14, 7, 19, 9 and 12 day for uncoated and coated apples with $100 \%$ Killer toxin, $100 \%$ Pullulan, 50\% Pullulan $+50 \%$ Killer toxin, $25 \%$ Killer toxin $+75 \%$ Pullulan and $75 \%$ Killer toxin $+25 \%$ Pullulan respectively.

\section{Conclusion}

Fruits coated with killer toxin that purified from Saccharomyces cerevisiae (bakery yeast) and Pullulan which produced from Aureobasidium pullulans lead to inhibition of microorganisms growth that is responsible for spoilage of fruits and it is very active for reduce weight loss in fruits during marketing which Leading to extending the shelf life of fruits.

\section{Acknowledgements}

None.

\section{Conflict of interest}

Author declares that there is no conflict of interest.

\section{References}

1. Alsoufi MA, Aziz RA. Use killer toxin extracted from bakery yeast for extending shelf life of fruits. Pak J Biotechnol. 2017;14(1):23-27.

2. Krasniewska K, Gniewosz M, Synowiec A, et al. The use of pullulan coating enriched with plant extracts from Satureja hortensis L. to maintain pepper and apple quality and safety. Postharvest Biol Technol. 2014;90:63-72.

3. Al-Soufi MA. Extending the storage life of some fruits by using Pullulan produced from locally isolate Aureobasidium pullulans. Iraqi J Mar Res Cons Protec. 2015;7(1):179-198.

4. Chlebowska-Smigiel A, Gniewosz M, Swinczak E. AN attempt to apply a pullulan and pullulan-proten coatings to prolong apples shelf-life stability. Acta Sci Pol Technol Aliment. 2007;6(1):49-56.

5. Gniewosz M, Synowiec A, Krasniewska K, et al. The antimicrobial activity of pullulan film incorporated with meadowsweet flower extracts (Filipendulae ulmariae flos) on postharvest quality of apples. Food Control. 2014;37(1):351-361. 
6. Cheng K-C, Demirci A, Catchmark J. Pullulan: biosynthesis, production, and applications. Appl Microbiol Biotechnol. 2011;92(1):29-44.

7. Mizrahy S, Peer D. Polysaccharides as building blocks for nanotherapeutics. Chem Soc Rev. 2012;41(7):2623-2640.

8. Chlebowska-Smigiel A, Gniewosz M. Effect of pullulan coating on inhibition of chosen microorganisms Growth. Acta Sci Pol Technol Aliment. 2009;8(3):37-46.

9. Shingel KI. Current knowledge on biosynthesis, biological activity, and chemical modification of the exopolysaccharide, pullulan. Carbohydr Res. 2004;339(3):447-460.

10. Singh RS, Saini GK, Kennedy JF. Pullulan: microbial sources, production and applications. Carbohydr Polym. 2008;73:515-531.

11. Gheorghe F, Marieta C, Paolo A. Preparation and characterization of $\mathrm{pH}$ and temperature-sensitive pullulan microspheres for controlled release of drugs. Biomaterials. 2008;29(18):2767-2775.

12. Na K, Lee DH, Hwang DJ, et al. pH-Sensitivity and $\mathrm{pH}$-dependent structural change in polymeric nanoparticles of poly (vinyl sulfadimethoxine)-deoxycholicacidconjugate. Eur Polym J. 2006;42(10):2581-2588.

13. Rekha MR, Sharma CP. Blood compatibility and in vitro transfection studies on cationically modified pullulan for liver cell targeted gene delivery. Biomaterials. 2009;30(34):6655-6664.

14. Scomparin A, Salmaso S, Versan S, et al. Novel folated and non-folated pullulan bioconjugates for anticancer drug delivery. Eur J Pharm Sci. 2011;42:547-558.

15. Wooram P, Kyoung SK, Byoung-chan B, et al. Cancer cell specific targeting of nanogels from acetylated hyaluronic acid with low molecular weight. Eur J Pharm Sci. 2010;40:367-375.

16. Mishra B, Vuppu S, Rath $\mathrm{K}$. The role of microbial pullulan, a biopolymer in pharmaceutical approaches: A review. J App Pharm Sci. 2011;1(6):45-50.

17. Gao FP, Cai YY, Zhou J, et al. Pullulan acetate coated magnetite nanoparticles for hyper-thermia: preparation, characterization and In vitro experiments. Nano Res. 2011;3(1):23-31.

18. Gaur R, Singh R. Optimization of physico-chemical and nutritional parameters for pullulan production by a mutant of thermotolerant Aureobasidium pullulans in fed batch fermentation process. Afr $J$ Biotech. 2010;9(43):7322-7330.

19. Gaur R, Singh R, Gupta M, et al. Aureobasidium pullulans, an economically important polymorphic yeast with special reference to pullulan. Afr J Biotech. 2010;9(47):7989-7997.
20. Aziz RA, Al-Soufi MA, Ateia AM. Purification and Determination of some Proteins inhibitors properties that produced from bakery yeast and study their activity against some types of bacteria that cause diarrhea. First International Scientific Conference, Cihan University, Iraq; 2014.

21. Shareef AM, Al-Dabbagh ASA. Effect of probiotic (Saccharomyces cerevisiae) on performance of broiler chicks. Iraqi $J$ Vet Sci. 2009;23(1):23-29.

22. Badia R, Brufau MT, Guerrero-Zamora AM, et al. $\beta$-Galactomannan and Saccharomyces cerevisiae var. boulardii modulate the immune response against Salmonella enterica serovar typhimurium in porcine intestinal epithelial and dendritic cells. Clin Vac Immunol. 2012;19(3):368-375.

23. Tukmechi A, Andani HRR, Manaffar R, et al. Dietary administration of beta-mercaptans-ethanol treated Saccharomyces cerevisiae enhanced the growth, innate immune response and disease resistance of the rainbow trout, Oncorhynchus mykiss. Fish Shellfish Immunol. 2011;30(3):923-928.

24. Kelly CP, Pothoulakis C, LaMont JT. Clostridium difficile colitis. N Engl J Med. 1994;330(4):257-262.

25. Desreumaux P, Neut C, Cazaubiel M, , et al. Saccharomyces cerevisiae CNCM I-3856 reduces digestive discomfort and abdominal pain in subjects with irritable bowel syndrome: a randomized double blinded placebo controlled clinical trial, abstr OP-181, 18th United European Gastroenterology Week. Barcelona, Spain; 2010.

26. Premanathan M, Shakurfow FAA, Ismail AA, et al. Treatment of oral candidiasis (thrush) by Saccharomyces cerevisiae. Int J Med Med Sci. 2011;3(3):83-86.

27. Kanmani P, Lim ST. Synthesis and characterization of pullulan mediated silver nanoparticles and its antimicrobial activities. Carbohydrate Polymers. 2013;97:421-428.

28. Jittinan C, Krisana S, Pakpachong V. The development of innovative herbal extract oral film for the unpleasant mouth odour. Int J Inno Sci Res. 2013;4(2):265-268.

29. Wagner A, Hetman B, Kopacki M, et al. Laboratory effect of boni protect containing Aureobasidium pullulans (de Bary) arnoud in the control of some fungal diseases of apple fruit. Acta Agrobotanica. 2013;66(1):77-88.

30. Aziz RA, Al-Soufi MA, Ateia AM. Study of bakery yeast extract activity on some enteric bacteria that isolated from some hospital in Baghdad city. J Colle Bas Edu. 2014;20(82):143-166.

31. Conforti FD, Totty JA. Effect of three lipid/hydrocolloid coatings on shelf life stability of Golden Delicious apples. Int J Food Sci Technol. 2007;42(9):1101-1106. 